\title{
Optimal Approximation for Functions Prescribed at Equally Spaced Points
}

\author{
H. F. Weinberger ${ }^{1}$
}

(February 10, 1961)

\begin{abstract}
Explicit upper and lower bounds for the value $F(u)$ of a linear functional $F$ applied to a function $u(x)$ defined on the interval $0 \leq x \leq 1$ are given when $u$ is prescribed at the $N+1$ points $i / N, \quad i=0, \ldots ., N$, and a bound for the integral of $u^{[k] 2}$ is known. These bounds are optimal in the sense that they are attained for functions satisfying the prescribed conditions. Their computation involves the inversion of a matrix of size $k-1$ rather than $N$, which means that $N$ is permitted to be large.
\end{abstract}

\section{Introduction}

Many problems in numerical analysis can be reduced to approximating the value $\vec{F}(u)$ of a given linear functional $F$ operating on an unknown element $u$ of a linear vector space. The approximation is to be made in terms of a inite set of data concerning $u$. Thus, the values $F_{1}(u), \ldots, F_{n}(u)$ of $N$ linear functionals acting on $u$ may be given. For example, the $F_{i}(u)$ may be values of the function $u$ at certain points $x_{i}$. If $F(u)$ is the value of $u$ at another point $\xi$, we have the problem of linear interpolation. If $F(u)$ is an integral of $u$, we have the problem of numerical quadratures. If $\mathrm{F}(u)$ is the value of a derivative, we have numerical differentiation.

It was shown by M. Golomb and the author ${ }^{2}$ that in order to obtain a finite interval in which the value $F(u)$ must lie, one must be given the value of at least one nonlinear functional operating on $u$. The simplest case is that in which one is given a bound for a quadratic functional $(u, u)$. In this case Golomb and the author ${ }^{2}$ showed how to obtain the exact interval in which $F(u)$ must lie when the values $F_{1}(u), \ldots, F_{N}(u)$, and $(u, u)$ are given. That is, upper and lower bounds for $F(u)$ which are attained for some elements $u$ satisfying the given conditions are found. The construction of these bounds requires the inversion of a matrix depending upon the functionals $F, F_{1}, \ldots, F_{N}$, and $(u, u)$.

In this paper we restrict our attention to a very simple case. We deal with a function $u(x)$ of a single variable on the interval $[0,1]$. The given functionals $F_{i}$ are the values $u(i / N)$ of $u$ at the $N+1$ equally spaced points $i / N, i=0, \ldots, N$. The quadratic functional is taken to be the integral of the square of the $k^{\text {th }}$ derivative of $u$.

We think of the number of points $N$ as large, while the number $k$ of the derivative will usually be small, say two or three. The matrix to be inverted is of size $N$. By making use of the equal spacing of our

1 Present address: University of Minnesota, Minneapolis, Minn.

${ }^{2}$ M. Golomb and H. F. Weinberger, Optimal approximations and error bounds, Symp. on Numer. Approx. (Univ. Wisconsin Press, Madison, Wis., 1959). points, we shall reduce its inversion to that of a $(k-1) \times(k-1)$ matrix. Thus the problem of obtaining best formulas for interpolation, quadratures, and numerical differentiation is made manageable even when the number of points involved becomes large.

When $F(u)=\int_{0}^{1} u d x$, our results yield as special cases the best quadrature formulas of Sard ${ }^{3}$ for $k \leq 3, N \leq 6$.

\section{Approximation Problem}

Let the values of the unknown function $u(x)$ be given at the $N+1$ evenly spaced points $i / N$, $i=0, \ldots, N$. Let $M^{2}$ be a given bound for the integral of the $k$ th derivative of $u$.

$$
(u, u)=\int_{0}^{1} u^{[k] 2} d x \leq M^{2} .
$$

We assume that $N \geq 2 k, \quad k \geq 2$.

Our problem is to approximate the value $F(u)$ of a certain linear functional $F$ applied to $u$. According to the theory in footnote 2 , this is possible if and only if the functional $F$ is bounded in the norm (2.1) for functions vanishing at the points $i / N$. That is, we must assume that there is a constant $c$ such that

$$
F(v)^{2} \leq c \int_{0}^{1} v^{[k] 2} d x
$$

for all $k$ times differentiable functions $v(x)$ such that

$$
v\left(\frac{i}{N}\right)=0, \quad i=0, \ldots, N .
$$

Any linear combination of pointwise values or integrals of $v$ and its derivatives up to order $k-1$ will satisfy this condition.

${ }^{3}$ A. Sard, Best approximate integration formulas; best approximation formulas, Am. J. Math. $\mathbf{7 1}, 80-91$ (1949). 
To obtain a best estimate for $F(u)$ we construct an auxiliary function $\bar{u}$ defined by the properties

$$
\bar{u}^{[2 k]}(x)=0, \quad 0<x<1, \quad N x \neq 0,1, \ldots, N
$$

and

$$
\begin{array}{ll}
\bar{u}\left(\frac{i}{N}\right)=u\left(\frac{i}{N}\right) & i=0,1, \ldots, N, \\
\bar{u}^{[l]}(0)=\bar{u}^{[l]}(1)=0 & l=k, \ldots, 2 k-2 .
\end{array}
$$

The function $u$ and its first $2 k-2$ derivatives are continuous, while $\bar{u}^{[2 k-1]}$ is allowed to have jump discontinuities at the points $i / N$.

The Green's function $G(x ; \xi)$ is defined by the properties

$$
\left.\begin{array}{ll}
\frac{\partial^{2 k} G}{\partial x^{2 k}}=0, & 0<x<1, N x \neq N \xi, 0,1, \ldots N, \\
G\left(\frac{i}{N} ; \xi\right)=0, & i=0,1, \ldots, N, \\
\frac{\partial^{l} G}{\partial x^{l}}=0, & \text { at } x=0,1, l=k, \ldots 2 k-2, \\
\frac{\partial^{2 k-1} G(\xi+0 ; \xi)}{\partial x^{2 k-1}}-\frac{\partial^{2 k-1} G(\xi-0 ; \xi)}{\partial x^{2 k-1}}=(-1)^{k} .
\end{array}\right\}
$$

Again $G$ and its first $2 k-2$ derivatives are continuous, while the $(2 k-1)$ st derivative may have discontinuities at $i / N$. By integration by parts we find that

$$
\bar{u}(\xi)=\sum_{i=0}^{N} g_{i}(\xi) u\left(\frac{i}{N}\right)
$$

where

$$
\begin{aligned}
g_{i}(\xi) & =(-1)^{k-1} \\
& \left\{\frac{\partial^{2 k-1} G\left(\frac{i}{N}+0 ; \xi\right)}{\partial x^{2 k-1}}-\frac{\partial^{2 k-1} G\left(\frac{i}{N}-0 ; \xi\right)}{\partial x^{2 k-1}}\right\} .
\end{aligned}
$$

Also by integration by parts we have

$$
u(\xi)-\bar{u}(\xi)=\int_{0}^{1}\left\{u^{[k]}-\bar{u}^{[k]}\right\} \frac{\partial^{k} G(x ; \xi)}{\partial x^{k}} d x .
$$

Applying the functional $F$ and using Schwarz's inequality, we find that

$$
\begin{aligned}
|F(u)-F(\bar{u})|^{2} & \leq \int_{0}^{1}\left|u^{[k]}-\bar{u}^{[k]}\right| 2 d x \int_{0}^{1} \mid F_{\xi}\left[\frac{\partial^{k} G(x ; \xi)}{\partial x^{k}}\right]^{2} d x \\
& =\int_{0}^{1}\left|u^{[k]}-\bar{u}^{[k]}\right|^{2} d x F_{\xi}\left\{F_{\eta}[G(\xi ; \eta)]\right\} .
\end{aligned}
$$

(The symbol $F_{\eta}[G(\xi ; \eta)]$ means that the functional $F$ is applied to $G$ considered as a function of $\eta$ for fixed $\xi . \quad F_{\xi}\left\{F_{\eta}[G(\xi ; \eta)]\right\}$ means that $F$ is then applied to the function $F_{\eta}[G]$ ). We have used the property

$$
G(\xi ; \eta)=\int_{0}^{1} \frac{\partial^{k} G(\xi ; x)}{\partial x^{k}} \frac{\partial^{k} G(\eta ; x)}{\partial x^{k}} d x,
$$

which follows from integration by parts.

Another integration by parts shows that

$$
\int_{0}^{1} \bar{u}^{[k]}\left\{u^{[k]}-\bar{u}^{[k]}\right\} d x=0 .
$$

Hence, we can rewrite (2.10) as

$$
|F(u)-F(\bar{u})|^{2} \leq\left\{M^{2}-\int_{0}^{1} \bar{u}^{[k]} d x\right\} F_{\xi}\left\{F_{\eta}[G(\xi ; \eta)]\right\} .
$$

Once $\bar{u}$ and $G$ are found, this inequality provides upper and lower bounds for $F(u)$. These bounds are sharp in the sense that we can construct functions $u$ satisfying (2.1) and having the given values $u(i / N)$ for which the bounds for $F(u)$ are attained.

We write $\bar{u}$ in the form

$$
\bar{u}(x)=\sum_{i=-k+1}^{N+k-1} a_{i} \Delta^{2 k}|N x-i|^{2 k-1} .
$$

The centered difference operator $\Delta^{2 k}$ is defined by

$$
\Delta^{2 k} c_{i}=\sum_{l=-k}^{k}\left(\begin{array}{c}
2 k \\
k+l
\end{array}\right)(-1)^{k+l} c_{i+l}
$$

It is easily seen that $\Delta^{2 k} \mid N x-i^{2 k-1}$ vanishes for $|N x-i| \geq k$. Hence the sum in (2.14) has at most $2 k-1$ nonzero terms.

Clearly the function (2.14) satisiies (2.4) and has the required continuity properties.

The coefficients $a_{i}$ are to be determined by the conditons (2.5). Thus we must have

$$
\sum_{i=-k+1}^{N+k-1} a_{i} \Delta^{2 k}|j-i|^{2 k-1}=u\left(\frac{j}{N}\right), \quad j=0,1, \ldots, N .
$$

In order to apply the last line of (2.5), we first use partial summation to write

$$
\bar{u}(x)=(-1)^{k} \sum_{i=-\infty}^{\infty} D^{k} a_{i} D^{k}|N x-i|^{2 k-i},
$$

where we have put $a_{i}=0$ for $i \leq-k, i \geq N+k$, and where $D^{k}$ is the $k$ th forward difference operator:

$$
D^{k} c_{i}=\sum_{l=0}^{k}\left(\begin{array}{l}
k \\
l
\end{array}\right)(-1)^{k+l} c_{i+l}
$$

Since $\Delta^{2 k} \mid N x-i^{2 k-1}=0$ for $|N x-i| \geq k$, (2.14) and (2.17) are independent of the values of $a_{i}$ for $i \leq-k$, $i \geq N+k$ when $0 \leq x \leq 1$. We note that $D^{k}|N x-i|^{2 k-1}$ 
is a polynomial of degree $k-1$ for $N x \leq i$ or $N x \geq i+k$. Hence the last line of (2.5) will be satisfied if

$$
\begin{aligned}
D^{k} a_{i}=0, \quad i= & -k+1, \ldots, \\
& -1, N-k+1, \ldots, N-1 .
\end{aligned}
$$

The boundary value problem (2.5) is thus replaced by the system (2.16), (2.19) of $N+2 k-1$ linear equations in the $N+2 k-1$ unknowns $a_{i}$.

We turn to this problem of matrix inversion. Remark: We have assumed that $k \geq 2$. The case $k=1$ is easily treated. Let $\bar{u}$ be the broken linear function coinciding with $\bar{u}$ at the points $i / N$ and having its breaks at these points. Let $G(x ; \xi)$ be the broken linear function with breaks at $\xi$ and the two neighboring points of the form $i / N$ which vanishes at the points $i / N$, and whose derivative decreases by -1 at $\xi$. Then (2.13) gives optimal bounds for $\dot{F}(u)$ when $k=1$.

\section{Matrix Inversion}

We consider the system of linear equations (2.16), (2.19). Since $\Delta^{2 k}|j-i|^{2 k-1}$ depends only upon $|j-i|$ and vanishes for $|j-i| \geq k, \quad(2.16)$ is a finite difference equation or order $2 k-2$. We solve it by means of a system of $2 k-2$ independent solutions of the homogeneous equation. To find these solutions, we note that for any number $z$

$$
\begin{aligned}
\sum_{i=-k+1}^{N+k-1} e^{i z} \Delta^{2 k}|j-i|^{2 k-1}=-2 e^{(j-k) z}\left(e^{z}-1\right)^{2 k} & \left(\frac{d}{d z}\right)^{2 k-1}\left[\left(e^{z}-1\right)^{-1}\right]
\end{aligned}
$$

for $0 \leq j \leq N$.

We define the polynomial in $e^{z}$

$$
Q_{l}\left(e^{z}\right) \equiv 2(-1)^{l+1} e^{-z}\left(e^{z}-1\right)^{l+2}\left(\frac{d}{d z}\right)^{l+1}\left[\left(e^{z}-1\right)^{-1}\right]
$$

It is easily seen to be of degree $l$ with leading coefficient 2 .

The $Q_{l}$ can be generated by the recursion

$$
\begin{aligned}
& Q_{l}(y)=(l y+1) Q_{l-1}(y)-y(y-1) Q_{l-1}(y), \\
& Q_{0}(y) \equiv 2
\end{aligned}
$$

The first few of these polynomials are

$$
\left.\begin{array}{l}
Q_{1}(y)=2(y+1), \\
Q_{2}(y)=2\left(y^{2}+4 y+1\right), \\
Q_{3}(y)=2\left(y^{3}+11 y^{2}+11 y+1\right), \\
Q_{4}(y)=2\left(y^{4}+26 y^{3}+66 y^{2}+26 y+1\right) .
\end{array}\right\}
$$

The coefficients of $Q_{2 k-2}$ are the coefficients of the finite difference equation (2.16). It can be shown by induction that they are symmetric in the sense that

$$
Q_{l}\left(\frac{1}{y}\right)=y^{-l} Q_{l}(y)
$$

This means that the zeros of $Q_{l}$ occur in reciprocal pairs. When $l$ is odd, one of the zeros is -1 . When $l$ is even, $Q$ can be written as a polynomial in $\left(y+y^{-1}\right)$. Thus, the zeros of $Q_{2 k-2}$ can be found by solving an equation of degree $k-1$ and a quadratic equation.

It is shown by induction that the coefficients of $Q_{l}$ are positive and that the zeros of $Q_{l}$ are real and negative. The zeros of $Q_{l}$ separate thos of $Q_{l+1}$.

Let $y_{1}<y_{2}<\ldots .<y_{2 k-2}<0$ be the zeros of $Q_{2 k-2}$ :

$$
Q_{2 k-2}\left(y_{\nu}\right)=0, \quad \nu=1, \ldots . ., 2 k-2 .
$$

Then by (3.7)

$$
y_{2 k-1-\nu}=\frac{1}{y_{\nu}} .
$$

Because of (3.1) and (3.2), the functions $a_{i}=y_{\nu}$ satisfy the homogeneous equations corresponding to (2.16). We now define

$$
\psi_{i}= \begin{cases}0 & i \leq k-2 . \\ \sum_{\nu=1}^{2 k-2} \frac{y_{\nu}^{i+k-2}}{Q_{2 k-2}^{\prime}\left(y_{\nu}\right)} & i \geq-k+2,\end{cases}
$$

It follows from the Lagrange interpolation formula [4] that the two definitions of $\psi_{i}$ coincide for $-k+2 \leq i \leq k-2$. Using (3.1) and (3.2), we find that

$\sum_{i=-k-1}^{N+k-1} \psi_{i-p} \Delta^{2 k}|j-i|^{2 k-1}=\delta_{j p}, \quad j, p=0, \ldots, N$.

The same equation is satisfied by $\psi_{i-p}$ plus any linear combination of the $y_{\nu}^{i}$. We add a linear combination such that the new function satisfies (2.19). Since $\psi_{i-p}$ vanishes for $i \leq p+k-2$ these conditions are already satisfied for $i=-k+1$, . . ., -2 . Therefore we add only functions which also satisfy these conditions. The Lagrange interpolation formula ${ }^{4}$ shows that such linear combinations are furnished by the functions $\eta_{i+\alpha}, \alpha=1, . . ., k$, where

Note that

$$
\eta_{i}=\sum_{\nu=1}^{2 k-2} \frac{y_{\nu}^{i+k-2}}{\left(y_{\nu}-1\right)^{k} Q_{2 k-2}^{\prime}\left(y_{\nu}\right)} .
$$

We let

$$
D^{k} \eta_{i}=\psi_{i}, \quad i \geq-k+2 .
$$

$$
\Gamma_{i p}=\psi_{i-p}-\sum_{\alpha=1}^{k} c_{\alpha} \eta_{i+\alpha}
$$

and determine the coefficients $c_{\alpha}$ in such a way that

${ }^{4}$ J. F. Steffensen, Interpolation (Chelsea Press, New York, N. Y., 1950. 
$D_{i}^{k} \Gamma_{i p}=0$ for $i=-1, N-k+1, \ldots ., N-1$. This gives $c_{k}=\delta_{0 p}$, and the equations

$\sum_{\alpha=1}^{k-1} c_{\alpha} \psi_{N-k+\alpha+\beta}=D^{k} \psi_{N-k+\beta-p}$

$$
-\delta_{0 p} \psi_{N+\beta}, \quad \beta=1, \ldots, k-1 .
$$

We let $A_{\alpha \beta}$ be the inverse of the symmetric $(k-1) \times(k-1)$ matrix $\psi_{N-k+\alpha+\beta}$ so that ${ }^{5}$

$$
\sum_{\beta=1}^{k-1} A_{\alpha \beta} \psi_{N-k+\beta+\gamma}=\delta_{\alpha \gamma}, \quad \alpha, \gamma=1, \ldots, k-1 .
$$

Then $\Gamma_{i p}$ is given by

$$
\begin{aligned}
& \Gamma_{i p}=\psi_{i-p}-\sum_{\alpha, \beta=1}^{k-1} A_{\alpha \beta} \eta_{i+\alpha} D^{k} \psi_{N-k-p+\beta} \\
&-\delta_{0 p}\left\{\eta_{i+k}-\sum A_{\alpha \beta} \eta_{i+\alpha} \psi_{N+\beta}\right\}, \quad i= \\
&-k+1, \ldots, N+k-1, \quad p=0, \ldots, N .
\end{aligned}
$$

This function satisfies

$$
\sum_{i=-k+1}^{N+k-1} \Gamma_{i p} \Delta^{2 k}|i-j|^{2 k-1}=\delta_{j p}, \quad j, p=0, \ldots, N
$$

and

$$
\begin{aligned}
D_{i}^{k} \Gamma_{i p}= & 0, \quad i=-k+1, \ldots,-1, \\
& N-k+1, \ldots, N-1 ; \quad p=0, \ldots, N .
\end{aligned}
$$

The solution of (2.16), (2.19) is given by

$$
a_{i}=\sum_{p=0}^{N} \Gamma_{i p} u\left(\frac{p}{N}\right) .
$$

Therefore $\Gamma_{i p}$ is the inverse matrix for the problem (2.16), (2.19).

Our problem of matrix inversion has thus been reduced to the solution of polynomial equations of degree $k-1$ and two in order to find the $y_{v}$ and the inversion of the $(k-1)$-dimensional symmetric matrix $\psi_{N-k+\alpha+\beta}$.

Example: We consider the case $k=2$. The zeros of $Q_{2}(y)$ are

$$
\begin{aligned}
& y_{1}=-2-\sqrt{3}, \\
& y_{2}=-2+\sqrt{3} .
\end{aligned}
$$

The function $\psi_{i}$ defined by (3.10) is

$$
\psi_{i}= \begin{cases}0 & i \leq 0, \\ \frac{\sqrt{3}}{12}\left(y_{1}^{-i}-y_{1}^{i}\right) & i \geq 0 .\end{cases}
$$

5 The fact that $\psi_{N-k+\alpha+\beta}$ is nonsingular follows from the uniqueness of $\bar{u}$ defined by (2.4), (2.5), and the linear independence of the functions $\Delta^{2 k}|N x-i|^{2 k-1}$ and $\eta_{i+\alpha}, \alpha=1, \ldots, k$.
Since $k-1=1$, the matrix $A_{\alpha \beta}$ has the single element $\psi_{N}^{-1}$. Moreover,

$$
\begin{aligned}
D^{2} \psi_{i} & =-\frac{1}{2} \sqrt{3}\left(y_{1}^{-i-1}-y_{1}^{i+1}\right), \quad i \geq 0 \\
D^{2} \psi_{-1} & =\frac{1}{2} \\
\eta_{i} & =-\frac{1}{72} \sqrt{3}\left(y_{1}^{1-i}-y_{1}^{i-1}\right) .
\end{aligned}
$$

Thus (3.17) gives

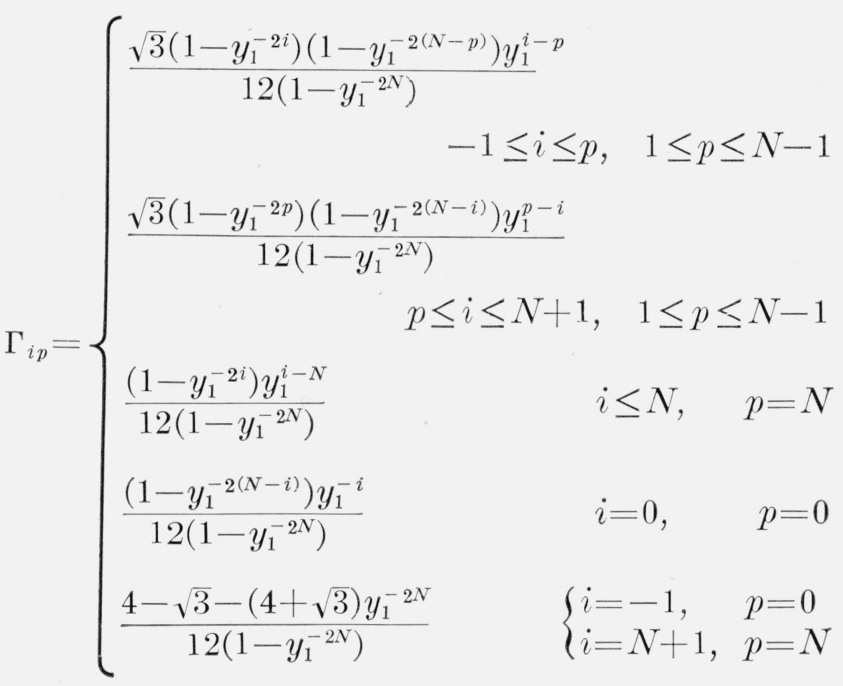

\section{Bounds}

We now return to the consideration of section 2 . The function $\bar{u}$ is given by

$$
\bar{u}(x)=\sum_{\substack{i=-k+1 \\|N x-i|<k}}^{N+k-1} a_{i} \Delta^{2 k}|N x-i|^{2 k-1},
$$

with

$$
a_{i}=\sum_{j=0}^{N} \Gamma_{i j} u\left(\frac{j}{N}\right)
$$

Thus, we may write

$$
\bar{u}(x)=\sum_{j=0}^{N} g_{j}(x) u\left(\frac{j}{N}\right),
$$

where

$$
g_{j}(x)=\sum_{\substack{i=-k+1 \\|N x-i|<k}}^{N+k-1} \Gamma_{i j} \Delta^{2 k}|N x-i|^{2 k-1}
$$

is the optimal approximation function corresponding to $u(i / N)=\delta_{i j}$.

In order to find the bounds (2.13) we need the integral of $\bar{u}^{[k] 2}$ and $G(x, \xi)$. Integrating by parts we find that

$$
\int_{0}^{1} \bar{u}^{[k]}{ }^{2} d x=(-1)^{k} \sum_{j=0}^{N} u\left(\frac{j}{N}\right)\left[\bar{u}^{[2 k-1]}\right]_{j / N},
$$


where []$_{j / N}$ denotes the discontinuity in the function at $j / N$, with the convention $\bar{u}^{[2 k-1]}(0-)=\bar{u}^{[2 k-1]}$ $(1+)=0$. Applying partial summation to (4.1), we find that

$$
\bar{u}(x)=\sum_{i=-k+1}^{N+k-1}|N x-i|^{2 k-1} \Delta^{2 k} a_{i} .
$$

Consequently,

$$
\begin{aligned}
& \int_{0}^{1} \bar{u}^{[k]} d x=(-1)^{k} 2 N^{2 k-1}(2 k-1) ! \sum_{i=0}^{N} u\left(\frac{i}{N}\right) \Delta^{2 k} a_{i} \\
&=(-1)^{k} 2 N^{2 k-1}(2 k-1) ! \\
& \sum_{i, j=0}^{N} u\left(\frac{i}{N}\right) u\left(\frac{j}{N}\right) \Delta_{i}^{2 k} \Gamma_{i \jmath}
\end{aligned}
$$

with the convention that

$$
\Delta^{2 k} \Gamma_{i j}=D^{k} \Gamma_{i j} \quad \text { for } \quad i=0, i=N .
$$

We now construct the Green's function $G(x, \xi)$. We begin with a function $H(x, \xi)$ having the proper jump at $x=\xi$ and satisfying the condition that the derivatives of orders $k, \ldots 2 k-2$ vanish at the end points. Let $p$ be any integer satisfying

$$
0 \leq p<N \xi<p+2 k-1 \leq N .
$$

It follows from the Lagrange interpolation formula (see footnote 4) that the function

$$
H(x ; \xi)=|x-\xi|^{2 k-1}-\sum_{\mu=0}^{2 k-1}\left|\frac{p+\mu}{N}-x\right|^{2 k-1} b_{\mu}(\xi),
$$

where

$$
\begin{aligned}
b_{\mu}(\xi)=\frac{(-1)^{\mu+1} N^{2 k-1}}{(2 k-1) !}\left(\begin{array}{c}
2 k-1 \\
\mu
\end{array}\right) & \left(\xi-\frac{p+\mu}{N}\right)^{-1} \prod_{\nu=0}^{2 k-1}\left(\xi-\frac{p+\nu}{N}\right)
\end{aligned}
$$

has the property

$$
H(x ; \xi) \equiv 0 \quad \text { for } \quad x \leq \frac{p}{N}, \quad x \geq \frac{p+2 k-1}{N} .
$$

Therefore, the function

$G(x ; \xi)=\frac{(-1)^{k}}{2(2 k-1) !}\left\{H(x ; \xi)-\sum_{j=0}^{N} g_{j}(x) H\left(\frac{j}{N} ; \xi\right)\right\}$

has all the properties (2.6). Thus, it is the Green's function. ${ }^{6}$ We not that the sums in (4.4) and (4.13) involve at most $2 k-1$ terms for each $x$ and $\xi$.

The bounds (2.13) for $F(u)$ are now given explicitly by

$$
\begin{aligned}
&\left|F(u)-\sum_{i=-k+1}^{N+k-1} \sum_{j=0}^{N} \Gamma_{i j} F\left(\Delta^{2 k}|N x-i|^{2 k-1}\right) u\left(\frac{j}{N}\right)\right|^{2} \\
& \leq \frac{(-1)^{k}}{2(2 k-1) !}\left\{M^{2}-2(-1)^{k} N^{2 k-1}(2 k-1) ! \sum_{i, j=0}^{N} \Delta_{i}^{2 k} \Gamma_{i j} u\left(\frac{i}{N}\right) u\left(\frac{j}{N}\right)\right\} \\
&\left\{F_{x}\left[F_{\xi}(H(x ; \xi))\right]-\sum_{i=-k+1}^{N+k-1} \sum_{j=0}^{N} \Gamma_{i j} F\left(\Delta^{2 k}|N x-i|^{2 k-1}\right) F\left[H\left(\frac{j}{N} ; \xi\right)\right]\right\}
\end{aligned}
$$

where we again use the convention (4.8). If $F$ is local in the sense that $F(u)$ only involves the values of $u$ in the neighborhood of a point, the sums in the second term on the right involve at most $2 k-1$ values of $i$ and $j$. Example: Letk=2. $\Gamma_{i j}$ is given by (3.22). We find that

$\Delta^{4}|N x-i|^{3}=\left\{\begin{array}{l}2\left\{4-6|N x-i|^{2}+3|N x-i|^{3}\right\} \\ 2\left\{2-|N x-i|^{3}\right\} \\ 0\end{array}\right.$

$$
\begin{aligned}
|N x-i| & \leq 1 \\
1 \leq|N x-i| & \leq 2 \\
|N x-i| & \geq 2 .
\end{aligned}
$$

Thus the interpolation function $g_{i}(x)$ is given by $g_{i}(x)=12(q+1-N x)(N x-q)\left[(q+2-N x) \Gamma_{q i}\right.$

$$
\begin{aligned}
& \left.\left.+(N x+1-q) \Gamma_{q+1},{ }_{i}\right]+(q+1-N x)^{3} \delta_{q \jmath}\right) \\
& +(N x-q)^{3} \delta_{q+1}, \jmath
\end{aligned}
$$

where the integer $q$ is defined by

$$
q<N x \leq q+1
$$

If $1<\mathrm{N} \xi<N-1$, we let $p$ be the integer such that

$$
p+1<N \xi<p+2 \text {. }
$$

${ }^{6}$ Since Green's function is uniquely defined by (2.6), it does not depend upon the integer $p$ used in the construction of $H$. The function $H$ does depend upon $p$. 


$$
\frac{3 N^{3} H(x ; \xi)}{(N \xi-p)(N \xi-p-1)(p+2-N \xi)(p+3-N \xi)}= \begin{cases}0 & N x \leq p, N x \geq p+3 \\ \frac{(N x-p)^{3}}{N \xi-p} & p \leq N x \leq p+1 \\ \frac{(N x-p)^{3}}{N \xi-p}-\frac{3(N x-p-1)^{3}}{N \xi-p-1} & p+1 \leq N x \leq N \xi \\ \frac{(p+3-N x)^{3}}{p+3-N \xi}-\frac{3(p+2-N x)^{3}}{p+2-N \xi} & N \xi \leq N x \leq p+2 \\ \frac{(p+3-N x)^{3}}{p+3-N \xi} & p+2 \leq N x \leq p+3 .\end{cases}
$$

If $0<N \xi<1$, the definition (4.18) gives $p=-1$, so that the function $H$ defined by (4.19) does not vanish at $x=0$. In this case we simply subtract the function

$$
\frac{1}{2}(N \xi+1)^{-1} \Delta^{4}|N x-1|^{3}
$$

from the right-hand side of (4.19) with $p=-1$ to obtain $H(x, \xi)$. Similarly, if $N-1<N \xi<N$, we subtract

$$
\frac{1}{2}(N+1-N \xi)^{-1} \Delta^{4}|N x-(N+1)|^{3}
$$

from the right-hand side of (4.19) with $p=N-2$.

Thus we can evaluate the bounds 4.14 explicitly. In the special case of linear interpolation we have

$$
F(u)=u(\zeta)
$$

If

$$
1 \leq q<N \zeta<q+1 \leq N-1
$$

we find

$$
\begin{aligned}
& F_{x}\left[F_{\xi}(H(x ; \xi))\right]-\sum_{i=-1}^{N+1} \sum_{j=0}^{N} \Gamma_{i j} F\left(\Delta^{4}|N x-i|^{3}\right) F\left[H\left(\frac{j}{N} ; \xi\right)\right] \\
& =N^{-3}(N \zeta-q)^{2}(q+1-N \zeta)^{2}\left\{4-\frac{\sqrt{3}}{3\left(1-y_{1}^{-2 N}\right)}\left[(q+2-N \zeta)^{2}\left(1-y_{1}^{-2 q}\right)\left(1-y_{1}^{-2(N-q)}\right)\right.\right. \\
& +2(q+2-N \zeta)(N \zeta-q+1)\left(1-y_{1}^{-2 q}\right)\left(1-y_{1}^{-2(N-q-1)}\right) y_{1}^{-1} \\
& \left.\left.+(N \zeta-q+1)^{2}\left(1-y_{1}^{-2(q+1)}\right)\left(1-y_{1}^{-2(N-q-1)}\right)\right]\right\},
\end{aligned}
$$

where

$$
y_{1}=-2-\sqrt{3} .
$$

The first factor on the right of (4.14) does not approach zero as $N \rightarrow \infty$ unless $M^{2}$ happens to be the exact value of the integral of $u^{[k] 2}$. Thus the difference between the best upper and lower bounds in the linear interpolation problem with $N+1$ equally spaced points and with a given bound for $\int_{0}^{1} u^{\prime \prime 2} d x$ is of the order $N^{-3 / 2}$. If a uniform bound for $\left|u^{\prime \prime}\right|$ is given, one can obtain bounds for $u(\zeta)$ which differ by a term of order $N^{-2}$. This shows that a bound for the square integral gives considerably less information than a bound for the maximum. The problem of finding best bounds when the maximum of $\left|u^{[k]}\right|$ is bounded is much more difficult than the problem treated here.

The author wishes to thank W. Börsch-Supan for a multitude of suggestions in connection with this paper.

(Paper 65B2-47) 